\title{
Interference in Coupled Structures with Strongly Un-balanced Phase Velocities of Normal Waves and its Electromagnetic Analogy with "Tsunami"
}

This paper was downloaded from TechRxiv (https://www.techrxiv.org).

\section{LICENSE}

CC BY 4.0

SUBMISSION DATE / POSTED DATE

$16-01-2022$ / 20-01-2022

\section{CITATION}

Malyutin, Nickolay; Loschilov, Anton; Malyutin, George; Sabitov, Timur (2022): Interference in Coupled Structures with Strongly Un-balanced Phase Velocities of Normal Waves and its Electromagnetic Analogy with "Tsunami". TechRxiv. Preprint. https://doi.org/10.36227/techrxiv.18481829.v1

$\mathrm{DOI}$ 


\title{
Interference in Coupled Structures with Strongly Un- balanced Phase Velocities of Normal Waves and its Electromagnetic Analogy with "Tsunami"
}

\author{
N. Malyutin, Member, IEEE, A. Loschilov, Member, IEEE, G. Malyutin, Timur Sabitov
}

\begin{abstract}
An analogy is drawn between the appearance of tsunami waves and "slow" waves in coupled structures (CS). Tectonic disturbances, as the cause of tsunami generation, generate "fast" waves similar to those in CS. The assumption is made that it is theoretically possible to give a rapid prediction of the occurrence of a natural disaster on the basis of information about the disturbing factor and environmental parameters. The paper notes the obvious problem of the lack of information about the parameters of the initial conditions of excitation of a rapid wave of tectonic origin in the prediction of tsunamis. It is possible that the considered electrodynamics analogy "Tsunami" will allow, firstly, to put and solve the problem of reconstruction of disturbing impact parameters, since the information about it comes before consequences, secondly, to develop "desktop" model of tsunami for some areas, thirdly, to develop measures to protect the coast by creating devices for diversion of tsunami wave energy part in safe directions.
\end{abstract}

Index Terms - Fast and slow waves, coupled structures, emitting pulses, electromagnetic analogy Tsunami, lack of information, disturbing factor, environmental parameters.

\section{INTRODUCTION}

$\mathrm{W}$ AVE propagation of different nature is studied in almost all fields of science and technology [1-3]. Wave energy transfer from barely perceptible low levels at nanowatt fractions [4-9], to several nanowatt fractions [4-9], and to several megawatts in a pulse [10-14], the movement of waves with enormous power during natural disasters such as tsunamis [15-20] are of great interest for experimental research, modeling and practical applications. Recently, interesting regularities in formation of the line segments response to an external pulse impact have been discovered and shown in the process of studying the features of interference of waves propagating in coupled strip lines with inhomogeneous dielectric filling [20]. Among such regularities is a relatively low level of reflections from the input, despite the fact that the frequency characteristics of the line segments have deep dips in the transmission coefficient in a wide range of frequencies. A more detailed study of the segments of coupled transmission lines, in which waves of in-phase and antiphase type can propagate, allowed us to discover the analogy between tsunami waves and waves having electromagnetic nature. The purpose of this paper is a description of this analogy with "Tsunami".

\section{COUPLED STRUCTURES}

The most common model of a real object, in which the formation of a waves propagating system with different phase velocities is observed, are coupled lines, the research of which is devoted many works. In the publications [22-26] on the basis of generalized telegraph equations solution an analytical expressions for calculation of waves propagating in coupled lines and having different phase velocities were obtained.

Further theoretical and experimental studies [27, 28] confirmed the validity of the quasi-T-wave theory for the purpose of designing a variety devices based on coupled lines. In the paper [22, 23] the system of eigenwaves (normal waves) is treated as a system of "even" and "odd" type waves propagating in the coupled lines with the same per unit parameters. Such waves are characterized by propagation coefficients $\gamma_{e}$ and $\gamma_{o}$. These coefficients are generally not equal, so when propagating "e" and "o" waves in coupled lines with different phase velocities, their vector addition occurs. Therefore, the phase shifts of waves "e" and "o" due to inequality $\gamma_{e}$ and $\gamma_{o}$ depend on the oscillation frequency, and in the coupled lines there is a frequency dependence of transmission and reflection coefficients [34-43].

Let us consider a system of two coupled strip lines. One of the possible designs of CS was proposed by [29, 30]. Figure 1, 2 shows a modification of such CS. The design consists of a vertically arranged substrate 1 , on which thin strips 2 of metal (copper) are applied, horizontally oriented substrate 4 has conductors 3 , on the reverse side of the substrate 4 there is a grounded plate 5 with a gap 6 parallel to conductors 2 .

A remarkable feature of the Figure 1 design is the ability to change the phase velocity of the waves excited in the coupled strips over a wide range. This property is shown in the paper [29] as well as in [30]. Let us illustrate this with the following examples of changes in the phase velocities of eigen-waves propagating in the $\mathrm{CS}$. The following design parameters were taken for the calculation (Figure. 2) $\varepsilon_{1}=\varepsilon_{2}=\varepsilon_{4}=1, \varepsilon_{3}=5$, $a=15, w_{1}=0, w_{2}=4, h_{21}=h_{2}=0.5, h_{3}=1.5, h_{4}=8$, $d=0$.

Figure 3, 4 show electric field patterns in the form of equi- 
$>$ REPLACE THIS LINE WITH YOUR MANUSCRIPT ID NUMBER (DOUBLE-CLICK HERE TO EDIT) <

potential lines calculated by the grid method [31] for even and odd excitation of bound strips. Comparison of the Figure 3 and Figure 4 graphs shows that when the strips are evenly excited, the electric field in substrate 1 is practically absent, all of its energy is concentrated in substrate 4 (see Figure 1).

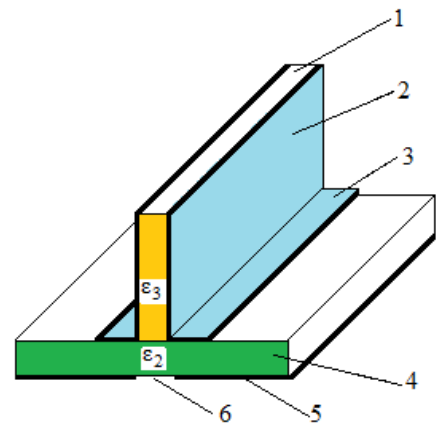

Fig. 1. Design of coupled strip lines on two dielectric substrates with relative permittivity's $\varepsilon_{2}$ and $\varepsilon_{3}\left(\varepsilon_{1}=1\right.$ air permittivity).

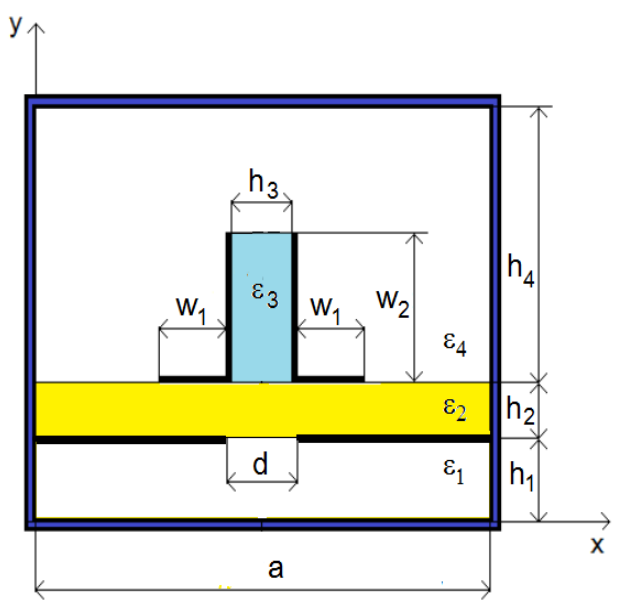

Fig. 2. Cross-sectional dimensions of the coupled strip lines used in the calculation of the primary parameters.

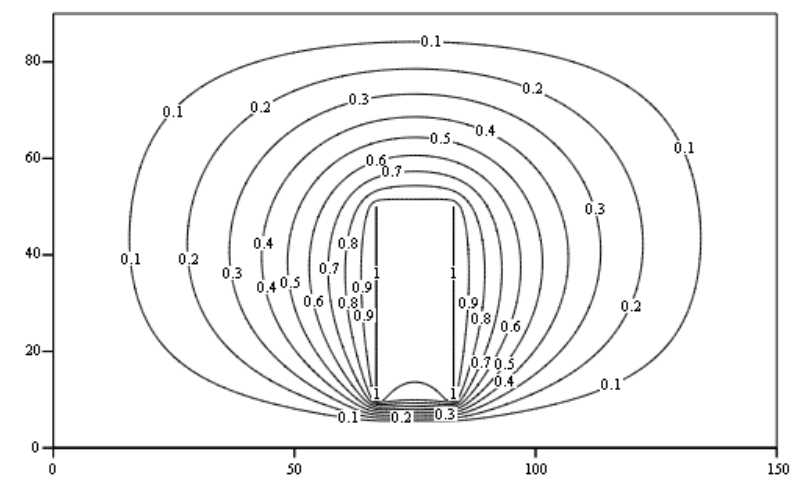

Fig. 3. Equipotential lines at even excitation of the coupled lines (potential on the strips $+1 \mathrm{~V}$ ).

With odd excitation, on the contrary, most of the energy of the electric field is concentrated in the substrate 1, the relative permittivity $\varepsilon_{3}$ of which is greater than the relative permittivity $\varepsilon_{2}$ of the substrate 4 . The ratio $\varepsilon_{2}$ and $\varepsilon_{3}$ incompletely determines the effective dielectric permittivities of the strip structure, since $\varepsilon_{1}$ and $\varepsilon_{4}$ also affect the coefficient of propagation of eigenwaves (even and odd waves).

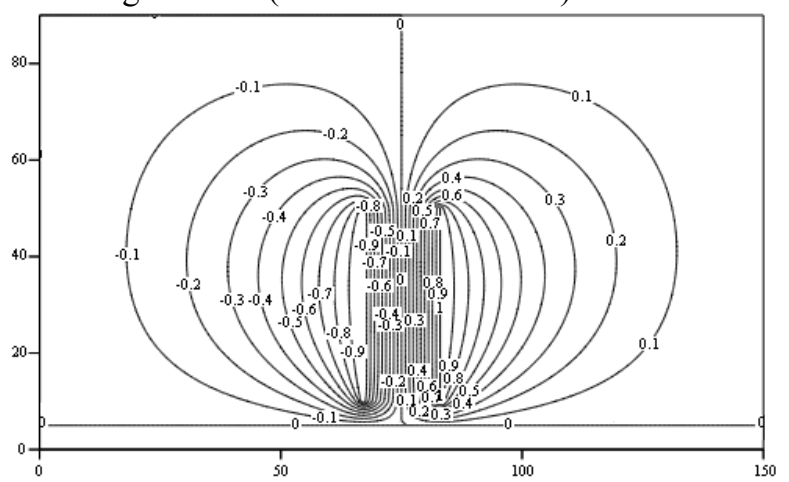

Fig. 4. Equipotential lines at odd excitation of bound lines (potential on the strips $(-1,+1) \mathrm{V}$ ).

The calculation shows that the effective permittivity for the even wave is $\varepsilon_{\text {effe }}=3.432$, and for the odd wave $\varepsilon_{\text {effo }}=1.184$ , i.e. both effective permittivities lie within the interval $(1,5)$. The phase velocities of the even mode and odd mode are $v_{e}=c / \sqrt{\varepsilon_{\text {effe }}}=2.756 \times 10^{8}, \quad v_{o}=c / \sqrt{\varepsilon_{\text {effo }}}=1.619 \times 10^{8}$ $\mathrm{m} / \mathrm{s}$, and theirs ratio is $v_{e} / v_{o}=1.702$.

\section{EIGEN AND BOUND WAVES}

Even and odd waves are eigenwaves. Their properties do not depend on the inclusion scheme of the coupled line segments, on their loads. Here there is an analogy with the definition of eigen frequencies of oscillating systems with lumped parameters. Therefore, the waves propagating in CSL with phase velocities $v_{e}, v_{o}$ can be called eigen or normal waves. But it should be remembered that their existence is possible only under certain boundary conditions, both in amplitude and phase [27]. A change in these conditions entails a transfer of energy from one line to another depending on the longitudinal coordinate. In this case bound waves are formed. Eigenwaves can propagate without reflection only when the lines are perfectly matched to even and odd type wave impedances, $Z_{0 e}$ and $Z_{0 o}$. In the example given, this is $Z_{0 e}=101.767 \mathrm{Ohms}$, $Z_{0 o}=24.79$ Ohms. The matching of bound waves at unequal phase velocities of in-phase and anti-phase waves is usually more complicated [27, 28]. Violation of excitation and matching conditions of eigenwaves leads to a change in the ratio of amplitudes and initial phase shifts of the excited waves. The picture of bound waves becomes more complex, since it is already determined by the superposition of incident and reflected waves propagating with different phase velocities $v_{e}$ and $v_{o}$, and, respectively, with different phase shifts.

In the work [21] the dependences of normal wave amplitudes on excitation type, value of loads and frequency were obtained. Calculation of frequency dependence of normal wave amplitudes of coupled lines with the above design di- 
$>$ REPLACE THIS LINE WITH YOUR MANUSCRIPT ID NUMBER (DOUBLE-CLICK HERE TO EDIT) <

mensions was carried out at different types of excitation of coupled lines according to the formulas of works [27]. Changing the excitation nature was carried out by varying the $E 1$ and $E 2$ (Figure 5) in such combinations:

$E 1=E 2=1-$ even type, also called in-phase type;

$E 1=1, E 2=-1-$ odd or antiphase type of excitation;

$E 1=1, E 2=0-$ combination type of excitation.

The loads in all ports are $50 \mathrm{Ohms}$.

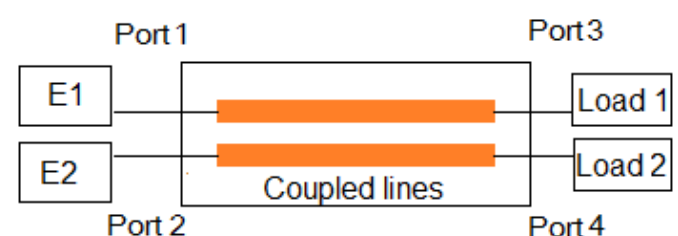

Fig. 5. Diagram of the coupled lines excitation.

The matrixes of per unit CSL's capacitances and inductances where determined numerically by solving the Laplace equation [31].

$$
\begin{aligned}
C & =\left[\begin{array}{cc}
142.41 & -106.71 \\
-106.71 & 142.41
\end{array}\right], \mathrm{pF} / \mathrm{m}, \\
L & =\left[\begin{array}{cc}
0.2611 & 0.1081 \\
0.1081 & 0.2611
\end{array}\right], \mu \mathrm{H} / \mathrm{m} .
\end{aligned}
$$

Figures 6-8 show the dependences of the amplitudes of the incident and reflected components of normal waves:

$A e$ - amplitude of the incident even (in-phase) wave;

$A o$ - amplitude of the incident odd (antiphase) wave;

$D e$ - amplitude of the reflected even (in-phase) wave;

Do - amplitude of the reflected odd (antiphase) wave.

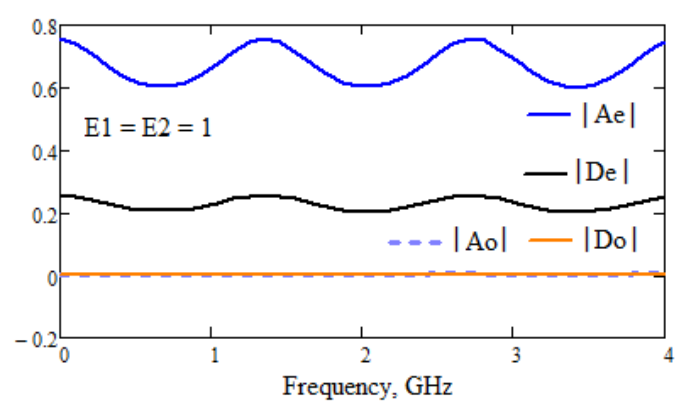

Fig. 6. Frequency dependence of normal wave amplitudes at even (in-phase) excitation of coupled lines.

The analysis of dependencies $A e, A o, D e, D o$ on the type of excitation shows that at even (in-phase) excitation in the lines (Figure 6) predominantly a wave with phase velocity $v_{e}=c / \sqrt{\varepsilon_{\text {effe }}}=2.756 \times 10^{8} \mathrm{~m} / \mathrm{s}$ propagates. Along with the incident component of amplitude $A e$ there is a reflected wave of amplitude $D e$, which is caused by mismatching of lines at wave impedance of even type $Z_{0 e}=101.7 \mathrm{Ohm}$ with loads $50 \mathrm{Ohm}$. At the same time the amplitudes of odd type waves $A o$ and $D o$ are close to zero.

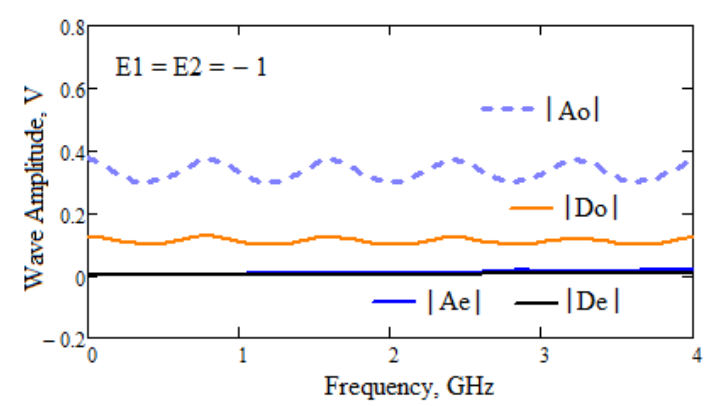

Fig. 7. Frequency dependence of normal wave amplitudes at odd (antiphase) excitation of coupled lines.

Figure 7 illustrates the picture of the wave process with odd (antiphase) excitation of the coupled lines. We see wave propagation with $A o$ and $D o$ amplitudes at a lower phase velocity of $v_{o}=c / \sqrt{\varepsilon_{\text {effo }}}=1.619 \times 10^{8} \mathrm{~m} / \mathrm{s}$. In this excitation variant, waves with amplitudes $A e, D e$ practically do not participate in energy transfer, since their amplitudes are close to zero.

At $E 1=1, E 2=0$, we observe (Figure 8) a more complex picture of the wave process components. We see the simultaneous occurrence of even and odd components with comparable amplitudes, but their frequency dependence is significantly different, since the phase velocity ratio $v_{e} / v_{o}=1.7$.

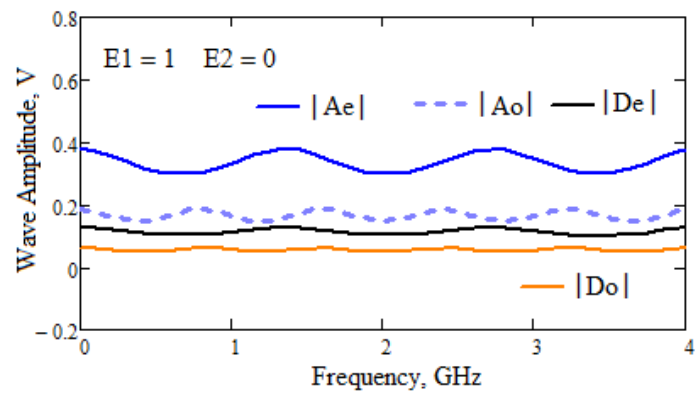

Fig. 8. Frequency dependence of normal wave amplitudes during combined excitation of coupled lines.

This is the reason for the standing waves formation, not only due to the superposition of incident and reflected waves, but also the superposition of incident waves of two types moving with different phase velocities $v_{e}$ and $v_{o}$.

In all cases considered, we did not observe the propagation of "pure" in-phase and antiphase waves, because the conditions of ideal harmonization of the wave components with amplitudes $A e, A o, D e, D o$, were not met. Theoretically, if this condition holds, the ratio of the amplitudes of the "pure" wave types with the same lines for in-phase excitation will be 1 , and for antiphase -1 , as it follows from [27, 28]. From Figure 6-8 this does not follow. Therefore, with respect to the considered wave excitation variants, it is reasonable to characterize quasi-T-wave propagation in the coupled lines as a process of bonded wave's emergence. These waves are a superposition of eigen or normal waves, for which the conditions of 
$>$ REPLACE THIS LINE WITH YOUR MANUSCRIPT ID NUMBER (DOUBLE-CLICK HERE TO EDIT) <

their existence in "pure" form are not met, i.e. the conditions of excitation and matching, prescribed by the nature of these waves.

The considered features of wave propagation in coupled lines under the influence of harmonic signals are true for each of the harmonic components of wide band pulse signals. But the superposition of responses to spectral harmonic components is accompanied by a number of interesting particularities of pulse signals propagation in coupled lines depending on the $v_{e} / v_{o}$ ratio [21] due to the frequency dependency of normal wave amplitudes.

For the analysis and experimental studies a model of the device was made [44-46]. Next strip sizes and substrate parameters (see Figure 2) $w_{1}=0, w_{2}=2 \mathrm{~mm}, h_{1}=1 \mathrm{~mm}$; $h_{2}=0.5 \mathrm{~mm} ; \varepsilon_{1}=1, \varepsilon_{2}=15, d=0$ were taken, coupled lines' length is $l=100 \mathrm{~mm}$. The coupled lines are then placed into a U-shaped screen, ports 1-4 of the device supplied with coax-strip transitions.

Per unit parameters $C_{11}=C_{22}=287.0 \mathrm{pF} / \mathrm{m}$, $C_{12}=C_{21}=253.7 \quad \mathrm{pF} / \mathrm{m}, \quad L_{11}=L_{22}=0.3343 \quad \mu \mathrm{H} / \mathrm{m}$, $L_{12}=L_{21}=0.1445 \mu \mathrm{H} / \mathrm{m}$, wave impedances of synphase excitement are $Z_{0 e}=119.91 \mathrm{Ohms}$, and $Z_{0 o}=18.74 \mathrm{Ohms}$ for antiphase excitement were gotten. The effective dielectric permittivities for the synphase wave are $\varepsilon_{\text {effe }}=1.435$, and $\varepsilon_{\text {effo }}=9.240$ for the antiphase wave, which conditioned the ratio of synphase and antiphase phase velocities $v_{e} / v_{o}=2.537$. The frequency characteristics of the coupled lines of the presented design were measured on a vector circuit analyzer R426 manufactured by Micran JSC, and the calculation was done using articles [27].

While measuring the frequency characteristics, the connection scheme of the coupled lines, shown in Figure 9, was used. The coaxial-strip transitions at ports 2 and 4 were removed, since they did not provide the no-load mode at these ports. The shown scheme is all-pass at the same phase velocities of in-phase and antiphase waves [32,33]. The calculated and experimental frequency dependences of the transmission coefficient $\left|S_{31}\right|$ of the considered coupled lines having matrixes of per unit CSL's capacitances and inductances (1) and (2) are shown in Figure 10.

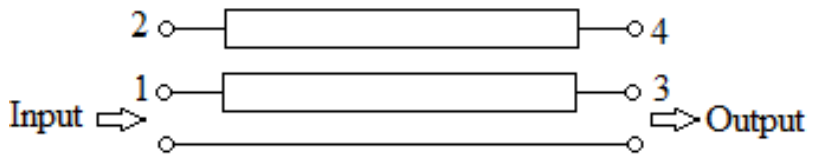

Fig. 9. Activation scheme of coupled lines during the research of frequency characteristics.

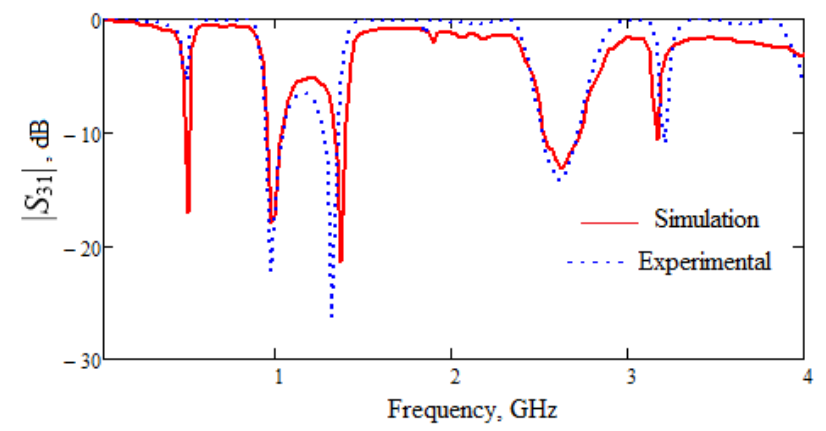

Fig. 10. Calculative and experimental dependencies of the transfer coefficient $\left|S_{31}\right|$ on the frequency.

As we can see, the use of coupled lines with a ratio of phase velocities of in-phase and antiphase waves $v_{e} / v_{o}=2.537$ drastically changes the frequency characteristics of the section on the CS.

\section{IMPULS PROPAGATION}

Experimental studies of pulse disturbances' propagation were done on a stand, the scheme of which is shown on Figure 11. The outer view of the used device of coupled strip lines pulse characteristics measurement is shown on Figure 12 [40]. The results of measurements are shown in Figure 13, 14. The pulse perturbation propagation was simulated by moving from the frequency domain to the time domain using the Fourier transform.

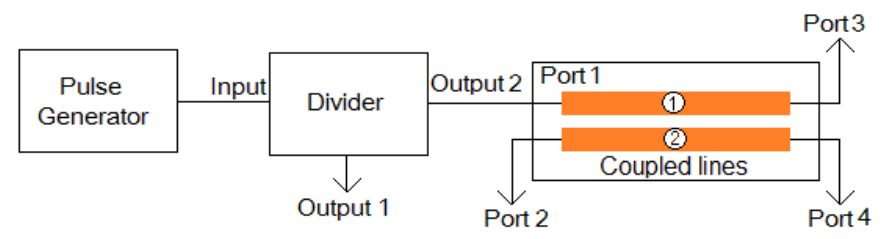

Fig. 11 The scheme for coupled strip lines pulse characteristics measurement.

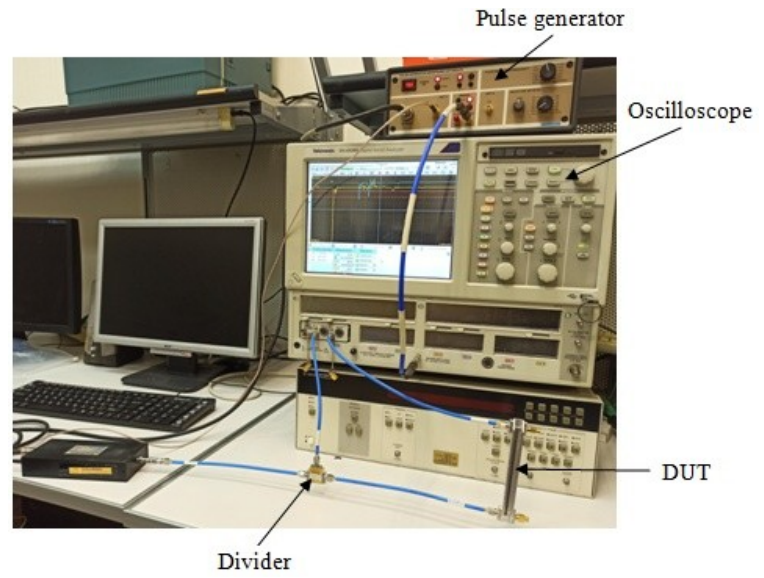

Fig. 12. Installation for impulse response measurement.

Figure. 13 shows the 40 ps pulse propagation from port 1 (line 1 input) to port 3 (line 1 output). The calculative dependency is highlighted in red, the experimental - in black. We can observe the appearance of several pulses of different amplitudes in port 3 on the output of the first line. It follows from 
the experimental dependencies of the graphs, that the second pulse is delayed relative to the first one, which arrived to port 3 for $\tau_{o}-\tau_{e}=0.655 \mathrm{~ns}$. The calculation shows $\tau_{o}-\tau_{e}=0.650 \mathrm{~ns}$, which indicates the adequacy of the applied mathematical model of wave propagation in connected lines with the considered properties. Figure 14 illustrates the experimental time dependences of the voltage at port 4 with open ports 2 and 3 . Here we also observe the excitation and propagation of waves with different group delay times, corresponding to the in-phase and antiphase waves velocities.

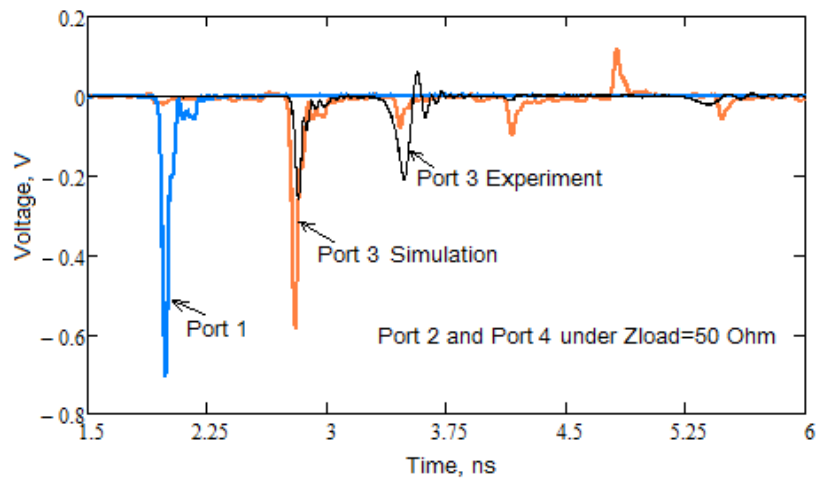

Fig. 13. Calculated and experimental dependences of pulse propagation with duration of $40 \mathrm{ps}$ from port 1 (input of line 1) to port 3 (output of line 1). Ports 2 and 4 are loaded with $50 \mathrm{Ohm}$ resistors.

The measurement scheme shown in Figure 11 allows measuring the voltage of the reflected wave from port 1 . This dependence was measured. Evaluation of the reflection coefficient $\Gamma$ from port 1 by comparing the maximum voltages of the input and reflected pulses showed the value $\Gamma \leq 0.2$. As a result, using the Fourier transform, the envelopes of the frequency spectra of the voltages at ports 1-4 and the voltage of the reflected wave were calculated.

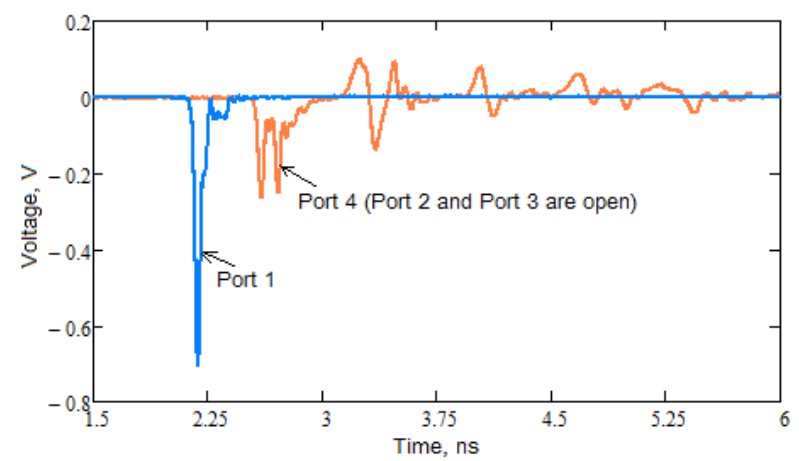

Fig. 14. Experimental voltage vs. time at port 4 when a 40 ps pulse is applied to port 1, ports 2 and 3 are unloaded (open).

The result of spectral analysis is shown in Figure 15. Analysis of the frequency dependence of spectral components shows that the energy of the excitation pulse, due to the inequality of phase velocities of in-phase and antiphase waves is transformed into the energies of mainly incident in-phase and antiphase waves. At the same time in ports 2 and 4 are observed close in amplitude spectral components of the same frequency, but in port 3 these components are absent.

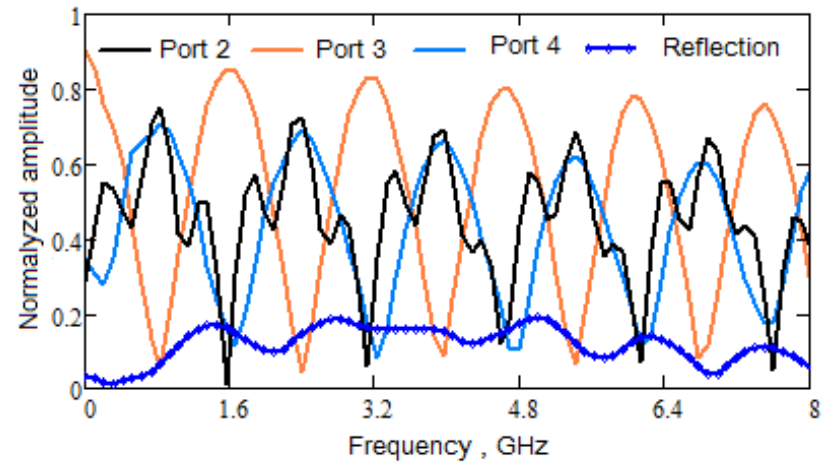

Fig. 15. The envelope spectra of the signals in ports $1-4$ and the envelope spectrum of the signal reflected from port 1 . All ports are loaded with $50 \mathrm{Ohms}$.

Conversely, the spectral components with minimum amplitude in ports 2 and 4 appear in port 3. This distribution of spectral components of in-phase and antiphase waves seems to indicate the formation of a system with two types of directivity, separated by some neutral zone.

\section{ANALOGY WITH "TSUNAMI"}

Tsunami waves have been the subject of many studies [15$20,47,48]$. The efforts of researchers are aimed at developing methods that make it possible to predict the nature and strength of a possible impact. Therefore, a significant part of the publications is devoted to the reconstruction of tsunamis that have already occurred. Knowing the source of wave excitation allows building mathematical models and calculating tsunami wave parameters. Exactly like this, the article [47] presents a way of modeling the soliton types waves in the ocean. Figure 16 from the article [47] illustrates tsunami waveforms obtained in result of observations and using numerical methods. Comparing Figure 16 and Figure 14, one can notice an outward similarity in the formation of "slow" waves occurring at time $t>3$ ns (Figure 14).

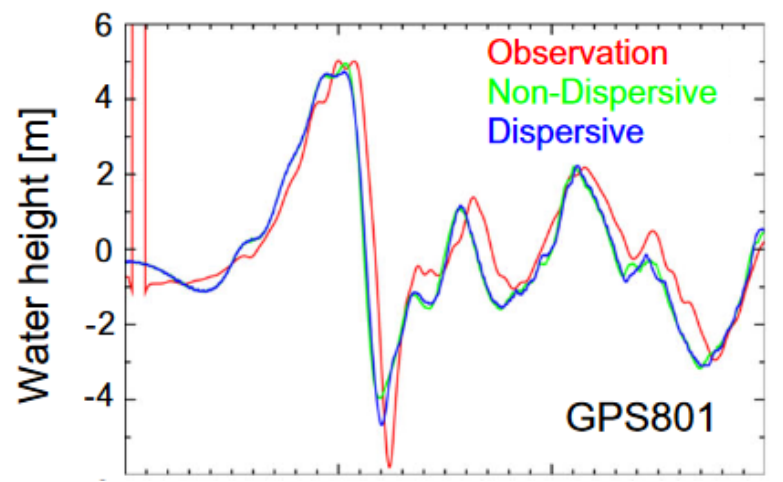

Fig. 16. Comparison of tsunami waveforms determined from observations (red), calculation based on the non-dispersion model (green), and calculation based on the dispersion model (blue). 
$>$ REPLACE THIS LINE WITH YOUR MANUSCRIPT ID NUMBER (DOUBLE-CLICK HERE TO EDIT) <

Note that these waves remotely resemble harmonic oscillations, although they are a superposition of harmonic responses to the spectral frequencies of external influence. Another important circumstance is that before the arrival of slowly propagating waves on the water perturbing oscillations of tectonic origin are registered [15-20].

Judging using the example of the considered electrodynamic analogue, this is a short pulse fed by the input of the first line (Figure 14, blue color) and the response to its impact at $\mathrm{t}<3 \mathrm{~ns}$. Due to the fact that the disturbing oscillations propagate in a solid medium with a much higher speed than oscillations in water, it is possible to obtain information about the nature of the disturbing impact before the arrival of the tsunami waves. This theoretically makes it possible to make a quick prediction of the occurrence of a natural disaster. Of course, an important parameter in this case is the coordinate of the tectonic shock occurrence point.

The modeling of wave propagation, including tsunami waves, is based on the physical features of wave phenomena in layered media with significantly different phase velocities. The authors [48] implemented a nested grid approach to numerical modeling for rapid calculation of tsunami wave propagation. A FPGA-based hardware acceleration unit was used to achieve high performance on a conventional modern PC. The numerical experiment is based on real digital bathymetry of northeastern Japan. The grid spacing varies from $276 \mathrm{~m}$ to $17 \mathrm{~m}$. A realistic shape of the initial sea surface displacement in the tsunami source was used. A similar approach to modeling the wave process was applied to the coupled line analysis [49].

\section{CONCLUSION}

From the analysis of mentioned and other articles on modeling tsunami there is an obvious problem of an information lack about quick wave of a tectonic or other origin excitement initial conditions parameters. It is possible that the considered electrodynamics' analogy "Tsunami" will allow, firstly, to put and solve the reconstruction problem of disturbing impact parameters, since the information about it comes before consequences, secondly, to develop "desktop" model of tsunami in occurrence relation to certain areas, thirdly, to develop measures to protect the coast by creating devices to withdraw part of the Tsunami wave energy in safe directions.

The presented results can be useful in the formulation and solving inverse problems of modeling reflections from targets for radar systems in an environment with different wave propagation coefficients [50]. Also, the described results of the study are of independent importance for understanding and modeling pulse propagation in electronics devices when protecting them from the destructive effects of powerful pulses, undistorted high-speed transmission of information over printed circuit board (PCB) conductors, design of frequency selection devices [51]. The particularities of wave interference in structures with significantly different phase velocities of normal waves, described in this paper, can have an analogy in integrated optics in the construction of electro-optical transducers and other devices containing inhomogeneous media.

\section{ACKNOWLEDGMEN}

This study was supported by the Ministry of Science and Higher Education Russian Federation, project no. FEWM2020-0039, March 1, 2020. The experimental studies were performed using the equipment of the "Impuls" Shared Access Center.

\section{REFERENCES}

[1] S.A. Schelkunoff, "Conversion of Maxwell's equations into generalized Telegraphist's equations." The Bell System Technical Journal. 34, pp. 995- 1043, 1995.

[2] J.R. Pierce, "Electronics, Waves and Messages." Hanover House, Garden City, New York, 1956 (Translation in Russian, 1961). 347 p.

[3] J. Pierce, "Almost all about waves." MIT Press Cambridge, Massachetusetts and London, 1974 (Translation in Russian). 177 p.

[4] A. Zagorodny, I. Yunusov., N. Drobotun, A. Drozdov and N. Voronin, "0.01-50 GHz power detector MMICs," in IEEE 15th Mediterranean Microwave Symposium (MMS). $30 \quad$ November 2015. Doi:10.1109/MMS.2015.7375486.

[5] V.T. Vo, and Z. Hu,. "Optimization and Realization of Planar Isolated GaAs Zero-Biased Planar Doped Barrier Diodes for Microwave/Millimeter-Wave Power Detectors/Sensors," IEEE Transactions on Microwave Theory and Techniques. Vol. 54, no 11, pp. 3836- 3842, 2006.

[6] A.S. Zagorodny, N.N. Voronin, I. Yunusov, and V.A. Gushchin, "Ultrawideband power detector GaAs MMIC's," in 15th International Conference of Young Specialists on Micro/Nanotechnologies and Electron Devices (EDM). 30 June, 2014. 2014, стр. 164-166. Doi: 10.1109/EDM.2014.6882503.

[7] Teresa M. Braun, "Low-Noise Amplifier and Frequency Converter. Chapter in book Satellite Communications Payload and System," WileyIEEE Press, 2012. Pp. 123 - 145. DOI: 10.1002/9781118345214.ch5.

[8] A.S. Zagorodny, A.V. Drozdov, N.N. Voronin, and I.V. Yunusov, "Modeling and application of microwave detector diodes." in 14th International Conference of Young Specialists on Micro/Nanotechnologies and Electron Devices, 1-5 July, pp. 96-99, 2013. Doi: 10.1109/EDM.2013.6641951.

[9] A.V. Khristenko, et al. Magnitude and Spectrum of Electromagnetic Wave Scattered by Small Quadcopter in X-Band. IEEE Transactions on Antennas and Propagation, 66, 1977- 1984 (2018).

[10]E.Balzovsky, Y.Buyanov, V. Koshelev, E. Nekrasov, V.Gubanov, A. Stepchenko, and A. Efremov, "A High-Power Source of Ultrawideband Radiation with Reflector Antenna," in Proceedings - 2018 20th International Symposium on High-Current Electronics, ISHCE 2018. 20, 80-84, 2018.

[11] Efremov, A.M., Koshelev, V.I., Plisko, V.V. \& Sevostyanov E.A. "A High-Power Synthesized Ultrawideband Radiation Source." Review of Scientific Instruments, 88, 094705, 2017.

[12]M.A. Samoylichenko, Yug. S. Zhechev, V. P. Kosteletskii and T.R. Gazizov, "Electrical Characteristics of a Modal Filter With a Passive Conductor in the Reference Plane Cutout." IEEE Transactions on Electromagnetic Compatibility. 63, 435-442 (2021).

[13]Rustam. R. Gazizov, Ruslan R. Gazizov, and Timur T. Gazizov, "The Detection of Voltage Extreme Points of the Shielded Power Supply Bus under the Ultrashort Pulse Excitation," in 21st International Conference of Young Specialists on Micro/Nanotechnologies and Electron Devices (EDM), 29 June-3 July, 2020.

[14]Rustam R. Gazizov, Ruslan R. Gazizov, and Timur T. Gazizov, "Simulation the Effect of Common-mode Excitation of Electrostatic Discharge on the Shielded Power Supply Bus Conductors." In Moscow Workshop on Electronic and Networking Technologies (MWENT). 11-13 March, 2020.

[15]J.Taniguchi, M. Miyoshi, T. Baba, and H. Aki, "A Numerical Simulation of Drainage of Influent Water Caused by Tsunami at Kawauchi, Tokushima." Techno-Ocean 2016: Return to the Oceans, pp. 489-492, 2017.

[16]S.-M. Jeong, J.-I. Park, and J.-C. Park, "Two-Dimensional Particle-Based Simulation of Tsunami-like Wave Run-up.” In OCEANS 2016 - Shanghai 
$>$ REPLACE THIS LINE WITH YOUR MANUSCRIPT ID NUMBER (DOUBLE-CLICK HERE TO EDIT) <

3 June, 2016, 7485505OCEANS 2016 - Shanghai, Shanghai, 122196 (2016).

[17]P. Lynett, and P. Lui, "Numerical Simulation of Complex Tsunami Behavior," Computing in Science \& Engineering. 13, 4, pp. 50 - 57, 2011.

[18]W.Windupranata, N. K. Suharjo, N.R. Hanifa, and C.A.D.S. Nusantara, "Reconstruction of the 2018 Palu Tsunami based on Tectonic Earthquake," in 2019 IEEE Asia-Pacific Conference on Geoscience, Electronics and Remote Sensing Technology (AGERS), 2019.

S.Chuai-Aree, and W. Kanbua, SiTProS: Fast and Real-Time Simulation of

Tsunami Propagation," in Proceedings - 1st Asia International Conference on Modelling and Simulation: Asia Modelling Symposium, 4148709, pp. 490495, 2007. Doi: 10.1109/ams.2007.95

[19]S.-M. Jeong, J.-I. Park, and J.-C. Park, “Two-Dimensional Particle-Based Simulation of Tsunami-like Wave Run-up." OCEANS 2016 - Shanghai 10-13 April, 2016.

A.N. Sychev, N.D. Malyutin, E.I. Trenkal and G.A. Malyutin, "Special aspects in interference of in-phase and anti-phase waves with unequal phase velocities in coupled lines under pulse impact." Journal of Physics: Conference Series, 1679, 2, 25 November 2020, 2nd International Scientific Conference on Applied Physics, Information Technologies and Engineering, APITECH, 2020, doi: 10.1088/1742-6596/1679/2/022023.

[20]E. Vlostovskiy, "Theory of coupled transmission lines," Telecommun. and Radio Engrg." 21, pp. 87-93, 1967.

[21]G.I. Zysman, and A.K. Johnson, "Coupled Transmission Line Networks in an Inhomogeneous Dielectric Medium." IEEE Transactions on Microwave Theory and Techniques 17. 10, pp. 753-759, 1969.

[22]J.L. Allen "Non-symmetrical coupled lines in an inhomogeneous dielectric medium." International Journal of Electronics 38, 337-347, 1975.

[23]V.K. Tripathi, "Asymmetric coupled transmission lines in an inhomogeneous medium." IEEE Trans. Microwave Theory Tech. 23, pp.734-739, 1975. Doi: $10.1109 /$ tmtt.1975.1128665.

[24]N.D. Malyutin, "Matrix parameters of non-identical, coupled striplines with a non-homogeneous dielectric." Radio Eng Electron Phys, ED-21, no. 12 , pp. 14-19, Dec. 1976.

[25]P.Vorob'ev, N. Malyutin and V. Fedorov. "Quasi-T-waves in devices using coupled strip lines with unbalanced electromagnetic coupling. "Radio Engineering and Electronic Physics (English translation of Radiotekhnika i Elektronika) ED-27, no. 9, pp. 38-44, Sep. 1982, issn 00337889 .

[26]N.D. Maljutin, A.G. Loschilov, A.N. Sychev and A.N. Maljutina. "Effect of multiple changes of phase and group velocity of traveling waves in the microwave broadband devices based on strip structures with a strongly unbalanced electromagnetic coupling," in Proceedings of Microwave \& Telecommunication Technology (CriMiCo), 24th International Crimean Conference, Sevastopol, Crimea, September 7-13, 2014, pp. 567-569.

[27]Y. Konishi, I. Awai, Y. Fukuoka, M. Nakajima, "A Directional Coupler of a Vertically Installed Planar Circuit Structure," IEEE Transactions on Microwave Theory and Techniques, ED-36, no. 6, pp, 1057-1063, 1988, doi 10.1109/22.3632.

[28]A.N. Sychev, S.M. Struchkov, N.Y. Rudyi, A.S. Salnikov, "Modeling of the vertically installed planar coupled lines by the numerical conformal transformation technique," in IEEE MTT-S Int. Conf. on Numerical Electromagnetic and Multiphysics Modeling and Optimization (NEMO), Seville,May 17-19, 2017, pp. 124-126, doi 10.1109/NEMO.2017.79642002.

[29]V.F. Fusco, Microwave circuits Analysis and Computer-aided Design. Prentice-Hall International, 1987 (Trans. into Russian), 87- 129 (1990).

[30]G. Matthaei, L. Young, T Jones, "Microwave filters, impedance matching networks and coupling structures," Norwood (MA): Artech House (1985).

[31]E. Jones, J. Bolljahn, "Coupled-strip-transmission line filters and directional couplers," in IRE Trans. Microwave Theory Tech., MTT-4, no. 2, pp.75-81, April 1956, doi: 10.1109/NVNN.1125022.

[32]B. A. Belyaev, A.M. Serzhantov, A.A. Leksikov, Y.F. Bal'va, E.O. Grushevskii, S.A. Khodenkov, "A Highly Selective Stripline Lowpass Filter with More than 100-dB Wide Stopband Attenuation," in Technical Physics Letters E-46, no. 4, pp. 364-367, April 2020, doi: $10.1134 / \mathrm{S} 1063785020040173$.

[33]G.M. Aristarkhov, N.V. Zvezdinov, "High-Selectivity single- and dualresonator microstrip filters," in Journal of Communications Technology and Electronics ED-62, no. 8, pp. 916-920, Aig. 2017, doi:10.1134/S1264226917080034.

[34] W. Ji, Y. Tong, Z. Zhang, W. Dai, Y. Li, "Dual wideband bandpass filter using multilayer broadside coupled structure," in Microwave and Optical Technology Letters, DE-62, no. 11, pp. 3451-3457, Novem. 2020, doi:10.1002/mop.32477.
[35]G.E. Cristal, "Coupled-transmission-line directional couplers with coupled lines of unequal characteristic impedances," in IEEE Microwave Theory and Technique, MTT-14, no. 7, pp. 337-346, 1966.

[36]S.B. Cohn, R. Levy, "History of microwave passive components with particular attention to directional couplers," in IEEE Trans. Microwave Theory and Technique, MTT-32, no. 9, pp. 1046-1054, Sept. 1984, doi: 10.1109/TMTT.1984.1132816.

[37]H. Liu, S. Fang and Z. Wang, "Trans-directional coupler with adjustable coupling coefficients and reconfigureurable responses," IET Microwaves, Antennas \& Propagation, 2017, vol. 11, no. 10, pp. 1340-1346.

[38]N. D. Malyutin, A. N. Malyutina, V. Fedorov, A. G. Loschilov and R. Sharabudinov, "Wave properties of the group delay correctors based on coupled strip-lines with different inphase and antiphase wave phase velocities," Journal of Electromagnetic Waves and Applications, 2019, vol 33, no. 3, pp. 367-318, doi: 10.1080/09205071.2018.1553688.

[39]A. O. Belousov, \& T. R. Gazizov, "Simulation of the time response in multiconductor microstrip modal filters with separate accounting for losses in conductors and dielectrics," Moscow Workshop on Electronic and Networking Technologies, 2018, Moscow, Russian Federation, Proceedings, pp. 1-5.

[40]R. A. Speciale, "Even-and odd-mode waves for nonsymmetrical coupled lines in nonhomogeneous media," IEEE Trans. on MTT MTT-23, 1975, vo1 1, pp. 897-908.

[41]V. R. Schlee, A. V. Efimenko and M. J. Voronin, "Relief coupled microstrip lines," Radiotechnics and Electronics, vol. 28 no. 6, pp. 10641071.

[42]A. N. Sychev, S. M. Struchkov et al., "A novel trans-directional coupler based on vertically installed planar circuit," in Proc. of the 45-th Eur. Microw. Conf., Paris, France, 2015, pp. 283-286. DOI: 10.1109/EuMC.2015.7345755.

[43] A. N. Sychev, S. M. Struchkov and N. Y. Rudyi, "A transdirectional coupled-line coupler with a vertical insert," in Microwave \& Telecommunication Technology, 2015, pp. 547-549.

[44]A. N. Sychev, S. M. Struchkov and N. Y. Rudyi, "Three-section transdirectional coupler of X-band," Proc. of the TUSUR, 2015 vol. 4 no. 36, pp. 12-16.

[45]T. Baba, N. Takahashi, Y. Kaneda, K. Ando, D. Matsuoka and T. Kato, "Parallel Implementation of Dispersive Tsunami Wave Modeling with a Nesting Algorithm for the Tohoku Tsunami," Pure and Applied Geophysics, 2015, vol. 172, no. 12, pp. 3455-3472.

[46]M. Lavrentiev, K. Lysakov, A. Marchuk, K. Oblaukhov, and M. Shadrin, "FPGA Based Modeling of Tohoku Tsunami Using Nested Grids," in Conference, Biloxi, Singapore, 2020, no. 0390177.

[47] N. D. Malyutin, A. N. Malyutina, and A. G. Loschilov, "Numerical Solution of Telegraph Equation Using Experimentally Determined Boundary Conditions for the Analysis of Waves in Strip Transmission Lines," in International Multi-Conference on Engineering, Computer and Information Sciences (SIBIRCON), Novosibirsk, Russian Federation, 2019, pp. 79-84, 10.1109/SIBIRCON48586.2019.8958140.

[48]T. Sabitov, A. Kiselev, M. Stepanov and M. Oreshkina, "Simulation of reflected signals in dual-position radar systems," in Department of Radio Receivers and Radio Transmitters, Novosibirsk, Russian Federation, 2021, vol. 12, no. 11, pp. 1082-1089, https://doi.org/10.1080/2150704X.2021.1964708.

[49]E. Chernikova., A. Belousov and T. Gazizov, "Analysis of Frequency Characteristics of a Reflection Symmetric Modal Filter," in International Multi-Conference on Engineering, Computer and Information Sciences (SIBIRCON), Novosibirsk, Russian Federation, 2019, pp. 212-216. 